Erratum: Polymer Journal, Vol. 40, No. 6, pp 534-537

\title{
Miniaturization of Surface Patterns by Combination of Contact Etching Lithography and Multi-step Shrinking of Stretched Polymer Films
}

By Hiroshi YABU ${ }^{1}$ and Masatsugu SHIMOMURA ${ }^{2}$

${ }^{1}$ Institute of Multidiciplinary Research for Advanced Materials, Tohoku University, 2-1-1 Katahira, Sendai 980-8577, Japan

${ }^{2}$ Advanced Institute for Materials Research, WPI, Tohoku University, 2-1-1 Katahira, Sendai 980-8577, Japan

Received: January 24, 2008

Accepted: February 27, 2008

Published: April 9, 2008

doi:10.1295/polymj.PJ2008022

On page 537, "Electronic Supporting Information Available" is added between "CONCLUSION" and "REFERENCES". 